\title{
Novel spiral mapping catheter facilitates observation of the time-to-pulmonary vein isolation during cryoballoon ablation
}

\author{
Alexander Pott ${ }^{1} \cdot$ Kerstin Petscher $^{1} \cdot$ Michael Baumhardt ${ }^{1} \cdot$ Tilman Stephan $^{1} \cdot$ Manuel Rattka $^{1} \cdot$ Rima Paliskyte $^{1}$. \\ Carlo Bothner ${ }^{1} \cdot$ Mirjam Keßler $^{1} \cdot$ Wolfgang Rottbauer $^{1} \cdot$ Tillman Dahme $^{1}$
}

Received: 8 April 2018 / Accepted: 31 August 2018 / Published online: 9 October 2018

(c) The Author(s) 2018

\begin{abstract}
Observation of the time-to-pulmonary vein isolation (TTI) by a spiral mapping catheter has emerged as a valuable procedural parameter in cryoballoon pulmonary vein isolation (PVI). The 1st generation spiral mapping catheter (Achieve, SMC1) has been available as an 8-polar catheter with a distal loop diameter of 15 or $20 \mathrm{~mm}$. The novel spiral mapping catheter (Achieve Advance, SMC2) was designed as a true guidewire and is available, in addition to the sizes of the SMC1, as a 10-polar mapping catheter with a distal loop diameter of $25 \mathrm{~mm}$. Whether these novel features of SMC2 influence procedural characteristics of Cryo-PVI in comparison to SMC1 has not been reported. In this prospective cohort study 158 patients (age $65.1 \pm 12.4$ years, female 39\%, paroxysmal AF 60\%) undergoing PVI with the 2nd generation cryoballoon were included. SMC1 was used in 57 patients (36\%), whereas 101 patients (64\%) underwent Cryo-PVI with the SMC2. All PVs (623/623, $100 \%$ ) were isolated successfully. Mean procedure duration was $72.0 \pm 18.9 \mathrm{~min}$ in the SMC1 group and $74.4 \pm 19.1 \mathrm{~min}$ in the SMC2 group $(p=0.432$ ). Mean fluoroscopy time was also not different between both study groups (SMC1 $15.7 \pm 6.6 \mathrm{~min}$, SMC2 $15.7 \pm 7.3 \mathrm{~min}, p=0.593$ ). TTI was observed in $68.6 \%$ of pulmonary veins in the SMC1 group, whereas TTI observation rate was $82.6 \%$ in the SMC2 group $(p<0.001)$. Number of freezes $(5.5 \pm 1.5$ vs. $6.5 \pm 1.9 ; p=0.001)$ and total freeze duration $(14.1 \pm 4.5$ vs. $17.6 \pm 5.6 ; p<0.001)$ were increased in the SMC2 group. SMC2 significantly increases TTI observation rate during Cryo-PVI. Procedure duration and fluoroscopy time are similar and number of freezes and total freeze duration are increased compared to PVI with SMC1 due to decreased stability and maneuverability of SMC2.
\end{abstract}

Keywords Atrial fibrillation · Cryoballoon · Spiral mapping catheter $\cdot$ Time-to-isolation $\cdot$ Achieve advance

\section{Introduction}

Pulmonary vein isolation (PVI) is the cornerstone in the treatment of patients with symptomatic atrial fibrillation [1-4]. During PVI, recording and evaluation of pulmonary vein $(\mathrm{PV})$ potentials significantly contributes to an effective ablation procedure [5]. Real-time observation of pulmonary vein isolation with a spiral mapping catheter (Achieve, Medtronic Inc, Minneapolis, MN, SMC1, Fig. 1a) placed in

Electronic supplementary material The online version of this article (https://doi.org/10.1007/s00380-018-1254-x) contains supplementary material, which is available to authorized users.

Tillman Dahme

tillman.dahme@uniklinik-ulm.de

1 Department of Medicine II, Ulm University Medical Center, Albert-Einstein-Allee 23, 89081 Ulm, Germany the pulmonary vein via the inner lumen of the cryoballoon catheter has emerged as a valuable procedural parameter to predict freedom from atrial fibrillation during cryoballoon PVI. A short time-to-isolation (TTI) is a predictor of low recurrence rates, whereas a long TTI is linked to high PV reconnection rates [6-8]. Furthermore, a high rate of TTI observation enables individual titration of cryoenergy and thereby reduces procedure duration and fluoroscopy time [9-13].

SMC1 has been available as an 8-polar catheter with a distal loop diameter of 15 or $20 \mathrm{~mm}$. The 2 nd generation of the spiral mapping catheter (Achieve Advance, Medtronic Inc, Minneapolis, MN, SMC2), has been designed as a true guidewire by abandoning the steel jacket of SMC1 which contained the electrical wires and instead putting the electrical wires around a core wire. This design changes lead to a much more flexible shaft. SMC2 is available, in addition to the sizes of the SMC1, as a 10-polar mapping catheter 


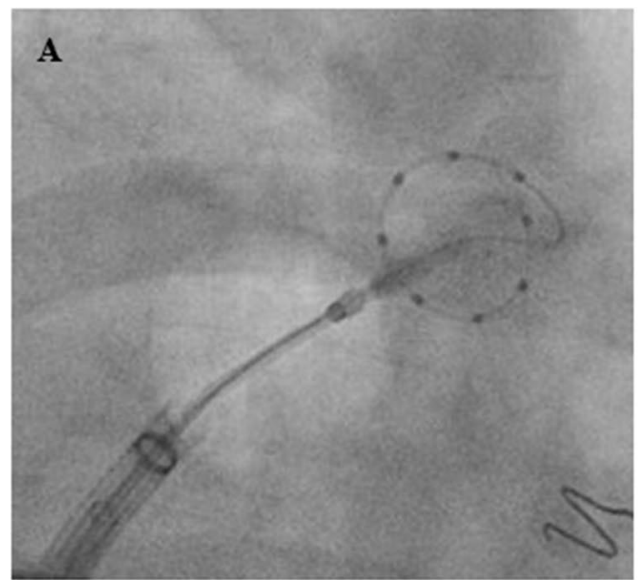

C

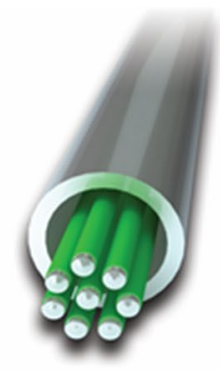

$\mathrm{SMC1}$

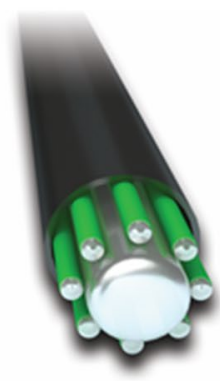

$\mathrm{SMC2}$

Fig. 1 Inflated arctic front advance cryoballoon with spiral mapping catheter introduced through the balloon inner lumen and positioned in the PV at the closest achievable proximity to enable real-time PV potential observation. a 1st generation 8-polar spiral mapping catheter (Achieve, SMC1) with $20 \mathrm{~mm}$ diameter. b 2nd generation 10-polar spiral mapping catheter (Achieve Advance, SMC2) with $25 \mathrm{~mm}$ diameter and 10 electrodes enables more proximal electrode

with a distal loop diameter of $25 \mathrm{~mm}$ (Fig. 1b-d). To date, no data on efficacy of PVI with SMC2 compared to SMC1 have been reported. This is to the best of our knowledge the first study evaluating the impact of SMC2 on cryoballoon $\mathrm{PVI}$ in comparison to SMC1.

\section{Methods}

\section{Study population}

In this prospective cohort study, we compared the first 41 patients, who underwent PVI for the treatment of paroxysmal or persistent atrial fibrillation with the 2 nd generation cryoballoon (Arctic Front Advance, Medtronic Inc, Minneapolis, MN, USA) in combination with the SMC2 to the last 101 patients treated with the 2 nd generation cryoballoon and SMC1. Exclusion criteria were long-standing persistent AF, previous left atrial (LA) ablation, LA diameter $>55 \mathrm{~mm}$, uncontrolled heart failure (NYHA class IV) and severe

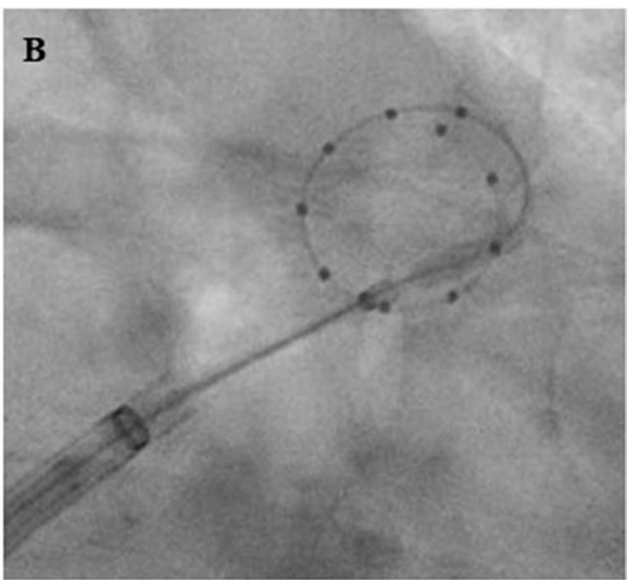

D

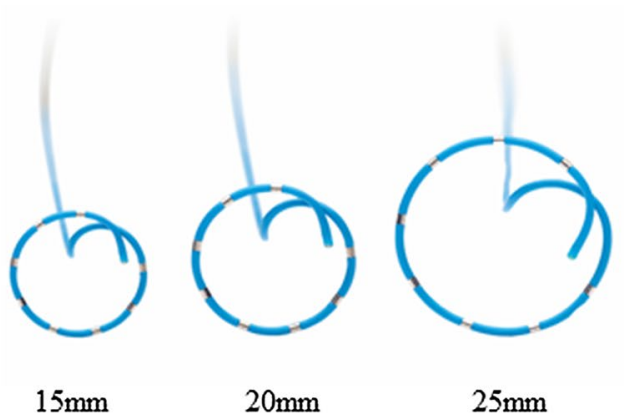

localization at the PV ostium. Fluoroscopic images recorded in RAO $30^{\circ}$. c The SMC1 was constructed with a steel jacket containing electrical wires. SMC2 was designed without a steel jacket, instead SMC2 was designed with a solid core wire and electrical wires are put around in a polyimite coating. d SMC1 and SMC2 are available as $15 \mathrm{~mm}$ and $20 \mathrm{~mm}$ diameter and 8 electrodes. SMC2 is available in addition as $25 \mathrm{~mm}$ diameter and with 10 electrodes

valvular disease. The study complies with the Declaration of Helsinki and was approved by our institutional review committee. All patients gave written informed consent to the procedure and to the participation in this observational study.

\section{Aim of the study}

We sought to evaluate the impact of SMC2 on procedure duration, fluoroscopy time, TTI observation rate and complication rate in patients during cryoballoon PVI in comparison to SMC1.

\section{Periprocedural management}

Intracardiac thrombi were ruled out in every patient by transesophageal echocardiography prior to PVI. Additional preprocedural imaging was not applied. Patients on vitamin $\mathrm{K}$ antagonists were scheduled for the procedure at a target INR of 2.0-3.0, whereas patients on non-vitamin $\mathrm{K}$ 
antagonist oral anticoagulants (NOACs) were advised to hold medication $24 \mathrm{~h}$ prior to the procedure. NOACs were continued on the evening of the day of the procedure. Anticoagulation was continued for at least 2 months following the procedure in all patients. Pericardial effusion was routinely ruled out by echocardiography immediately after the procedure and prior to hospital discharge.

\section{Cryoballoon ablation procedure}

Cryoballoon ablation with SMC1 has been described in detail before [9, 14]. Briefly, the cryoballoon was introduced to the left atrium via a steerable transseptal sheath (Flexcath Advance, Medtronic, Minneapolis, MN, USA) after single transseptal puncture. In the SMC2 group the selection of the diameter of the spiral mapping catheter (20 or $25 \mathrm{~mm}$ ) was at the operator's discretion in the first 41 cases based on PV diameter estimated by PV angiography. In the last 60 cases in the SMC2 group the $25 \mathrm{~mm}$ spiral mapping catheter was used exclusively. After balloon inflation and placement at the PV ostia, the spiral mapping catheter was positioned in the PV at the closest achievable proximity to the ostium to enable real-time observation of PV potentials during PV isolation. PV occlusion was documented by injection of contrast medium. The TTI was defined as the time of the last recording of a PV potential before sustained isolation. In both study groups individualized TTI-dependent titration of cryoenergy was performed. In case of a TTI $<30 \mathrm{~s}$ the total freeze cycle duration was decreased to $120 \mathrm{~s}$ and no bonus freeze was applied. If TTI was between 30 and $60 \mathrm{~s}$ a single $180 \mathrm{~s}$ freeze cycle was applied. If TTI was $>60 \mathrm{~s}$ a $180 \mathrm{~s}$ freeze cycle followed by a $180 \mathrm{~s}$ bonus freeze was applied. Also, if no TTI could be documented, but isolation was achieved, a single $180 \mathrm{~s}$ freeze cycle was applied followed by a $180 \mathrm{~s}$ bonus application of cryoenergy. Isolation of all PVs was reassessed at the end of the procedure by documentation of entrance- and exit-block except at PVs with a TTI $\leq 60 \mathrm{~s}$. Cryoenergy application was aborted if luminal esophageal temperature (LET) measured by transnasally inserted temperature probe (S-CATH; Circa Scientific Inc., Englewood, CO, USA) decreased below $20^{\circ} \mathrm{C}$ [15].

\section{Statistical analysis}

Significance of differences of numeric values between the two groups was calculated by $t$ test if normal distribution with equal variance was given. Normal distribution was determined by Shapiro-Wilk test and equal variance by Brown-Forsythe test. Numeric variables that were not normally distributed were analyzed by Mann-Whitney rank sum test. Categorical variables were analyzed by Chi-square test or Fisher's exact test. A $p$ value $<0.05$ was considered significant. Statistical assessment was performed with Excel
(Version 2016, Microsoft Inc., Redmond, WA, USA) or XLStat software (V 2016.02.28430, Addinsoft, New York, NY, USA).

\section{Results}

\section{Study population}

We included 158 patients (mean age $65.1 \pm 12.4$ years, female $39 \%$, paroxysmal AF 60\%, mean $\mathrm{CHA}_{2} \mathrm{DS}_{2}$-VASc-Score $2.8 \pm 1.6)$ undergoing cryoballoon PVI with the second generation cryoballoon. In 57 patients $(36 \%)$ the SMC1 was applied (SMC1 group) and 101 patients (64\%) underwent cryoballoon PVI with the SMC2 (SMC2 group). Baseline characteristics did not differ significantly between both study groups (Table 1). SMC1 was exclusively used with a diameter of $20 \mathrm{~mm}, \mathrm{SMC} 2$ with a $20 \mathrm{~mm}$ diameter was applied in 12 patients, SMC2 with a $25 \mathrm{~mm}$ diameter was used in 89 patients (Table 2 ).

\section{Procedural results}

In the SMC1 group we identified $226 \mathrm{PVs}$ and $397 \mathrm{PVs}$ were identified in the SMC2 group. All PVs (623/623, 100\%) were isolated successfully with the SMC1 or SMC2 placed in the lumen of the 2nd generation cryoballoon. Exchange of the mapping catheter to a stiff guidewire was necessary in one case in the SMC2 group. Overall TTI observation rate in the SMC1 group was $68.6 \%(155 / 226 \mathrm{PVs})$, whereas TTI observation rate for all PVs was $82.6 \%$ (328/397 PVs) in the SMC2 group $(p=0.001)$. We were able to determine the time-to-isolation in 46/55 LSPVs $(83.6 \%)$ in the SMC1 group and in $83 / 94$ LSPVs $(88.3 \%)$ in the SMC2 group $(p=0.696)$, in $32 / 55$ LIPVs $(58.2 \%)$ in the SMC1 group versus $73 / 94$ LIPVs $(77.7 \%)$ in the SMC2 group $(p=0.002)$, in $42 / 57$ RSPVs $(73.6 \%)$ in the SMC1 group compared to $81 / 101$ RSPVs $(80.2 \%)$ in the SMC2 group $(p=0.344)$ and in $35 / 57$ RIPVs (61.4\%) in the SMC1 group versus 84/101

Table 1 Patient baseline characteristics

\begin{tabular}{llll}
\hline & SMC1 & SMC2 & $p$ value \\
\hline Patients $(n)$ & 57 & 101 & \\
Gender, female $[n(\%)]$ & $25(43.8)$ & $37(36.6)$ & 0.469 \\
Age (years) & $64.1 \pm 11.3$ & $65.6 \pm 13.0$ & 0.303 \\
Paroxysmal Afib $[n(\%)]$ & $35(61.4)$ & $60(59.4)$ & 0.939 \\
$\mathrm{CHA}_{2} \mathrm{DS}_{2}$-VASc-Score & $2.8 \pm 1.5$ & $2.8 \pm 1.7$ & 0.714 \\
Heart failure $[n(\%)]_{\text {Arterial hypertension }[n(\%)]}$ & $21(36.8)$ & $37(36.6)$ & 0.884 \\
Diabetes mellitus $[n(\%)]$ & $8(14.0)$ & $70(69.3)$ & 0.690 \\
LA diameter $(\mathrm{mm})$ & $45 \pm 7$ & $44 \pm 8$ & 0.552 \\
\hline
\end{tabular}


Table 2 Procedural characteristics

\begin{tabular}{|c|c|c|c|}
\hline & SMC1 & SMC2 & $p$ value \\
\hline \multicolumn{4}{|l|}{ Mapping catheter size $[n(\%)]$} \\
\hline $20 \mathrm{~mm}$ (8 electrodes) & $57(100)$ & $12(12)$ & \\
\hline $25 \mathrm{~mm}$ (10 electrodes) & $0(0)$ & $89(88)$ & \\
\hline Procedure duration (min) & $72.0 \pm 18.9$ & $74.4 \pm 19.1$ & 0.432 \\
\hline Fluoroscopy time (min) & $15.7 \pm 6.6$ & $15.7 \pm 7.0$ & 0.593 \\
\hline PVs isolated $[n(\%)]$ & $226(100)$ & $397(100)$ & 1.000 \\
\hline Freezes per patient $(n)$ & $5.5 \pm 1.5$ & $6.5 \pm 1.9$ & 0.001 \\
\hline Freeze duration per patient (min) & $14.1 \pm 4.5$ & $17.6 \pm 5.6$ & $<0.001$ \\
\hline Freeze abortion $[n(\%)]$ & $4(1.3)$ & $32(4.9)$ & 0.010 \\
\hline PNP & 4 & 7 & 0.981 \\
\hline LET & 1 & 11 & 0.117 \\
\hline Balloon dislodgment & 0 & 4 & 0.314 \\
\hline Ineffectiveness & 0 & 9 & 0.036 \\
\hline Mean TTI all PVs (s) & $44.2 \pm 24.6$ & $45.9 \pm 27.4$ & 0.834 \\
\hline TTI LSPV & $49.9 \pm 25.8$ & $48.4 \pm 20.6$ & 0.532 \\
\hline TTI LIPV & $30.5 \pm 13.5$ & $36.9 \pm 18.4$ & 0.130 \\
\hline TTI RSPV & $40.6 \pm 18.1$ & $38.1 \pm 17.6$ & 0.627 \\
\hline TTI RIPV & $53.8 \pm 31.4$ & $56.1 \pm 40.5$ & 0.637 \\
\hline \multicolumn{4}{|l|}{ Complications $[n(\%)]$} \\
\hline Pericardial tamponade $[n(\%)]$ & $0(0)$ & $1(1)$ & 1.000 \\
\hline Groin hematoma $[n(\%)]$ & $1(1.8)$ & $0(0)$ & 0.361 \\
\hline Other $[n(\%)]$ & $0(0)$ & $0(0)$ & 1.000 \\
\hline
\end{tabular}

RIPVs (83.2\%) in the SMC2 group ( $p=0.004$, Fig. 2). No TTI was registered in 2 LCPVs in the SMC1 group, while in 3 out of 7 LCPVs a TTI was registered in the SMC2 group $(p=0.500)$.
We further analyzed the TTI observation rates with regard to the diameter of spiral mapping catheter in the 69 patients treated with a $20 \mathrm{~mm}$ catheter (SMC1 and SMC2) versus the 89 patients treated with a $25 \mathrm{~mm}$ catheter (only SMC2). While TTI observation rate with the $20 \mathrm{~mm}$ catheter was $70.2 \%$ (191/272 PVs), TTI was significantly more often registered with the $25 \mathrm{~mm}$ diagnostic catheter $(83.2 \%, 292 / 351$ PVs, $p<0.001)$, indicating that catheter size and additional electrodes facilitate registration of PV potentials during PV isolation. Accordingly, TTI observation rate between SMC1 (68.6\%) and $20 \mathrm{~mm}$ SMC2 (78.2\%) was statistically not different $(p=0.258)$. Comparison of TTI observation rate between SMC2 with $20 \mathrm{~mm}$ and $25 \mathrm{~mm}$ demonstrated a numerically higher real-time observation rate using a larger mapping catheter (20 mm: 78.2\% vs $25 \mathrm{~mm}$ : 83.2\%). However, this difference is statistically not significant $(p=0.407)$.

In contrast to the increased rate of observed real-time PV isolations with the SMC2 the actual time-to-isolation (TTI) did not differ between both groups. Mean time-to-isolation was $44.2 \pm 24.6 \mathrm{~s}$ in the SMC1 group and $45.9 \pm 27.4 \mathrm{~s}$ in the SMC2 group $(p=0.834)$. In accordance, there was also no significant difference in the mean TTI of each PV between both study groups.

However, incidence of prematurely aborted freezes caused by phrenic nerve palsy (PNP), low oesophageal temperature (LET) $<20^{\circ} \mathrm{C}$, cryoballoon dislodgment or ineffective pulmonary vein isolation was significantly higher when the 2nd generation spiral mapping catheter was used (SMC1: 4/314 freezes (1.3\%) vs. SMC2: $32 / 655$ freezes $(4.9 \%$; $p=0.009$ ). Especially, ineffective pulmonary vein isolation leading to premature freeze abortion was observed more
Fig. 2 Overall TTI observation rate and per vein depending on spiral mapping catheter. Using the SMC2 leads to significantly higher overall TTI observation rate and in the LIPV and RIPV, whereas TTI observation rate of the upper PV was comparable in the SMC1 and SMC2 group

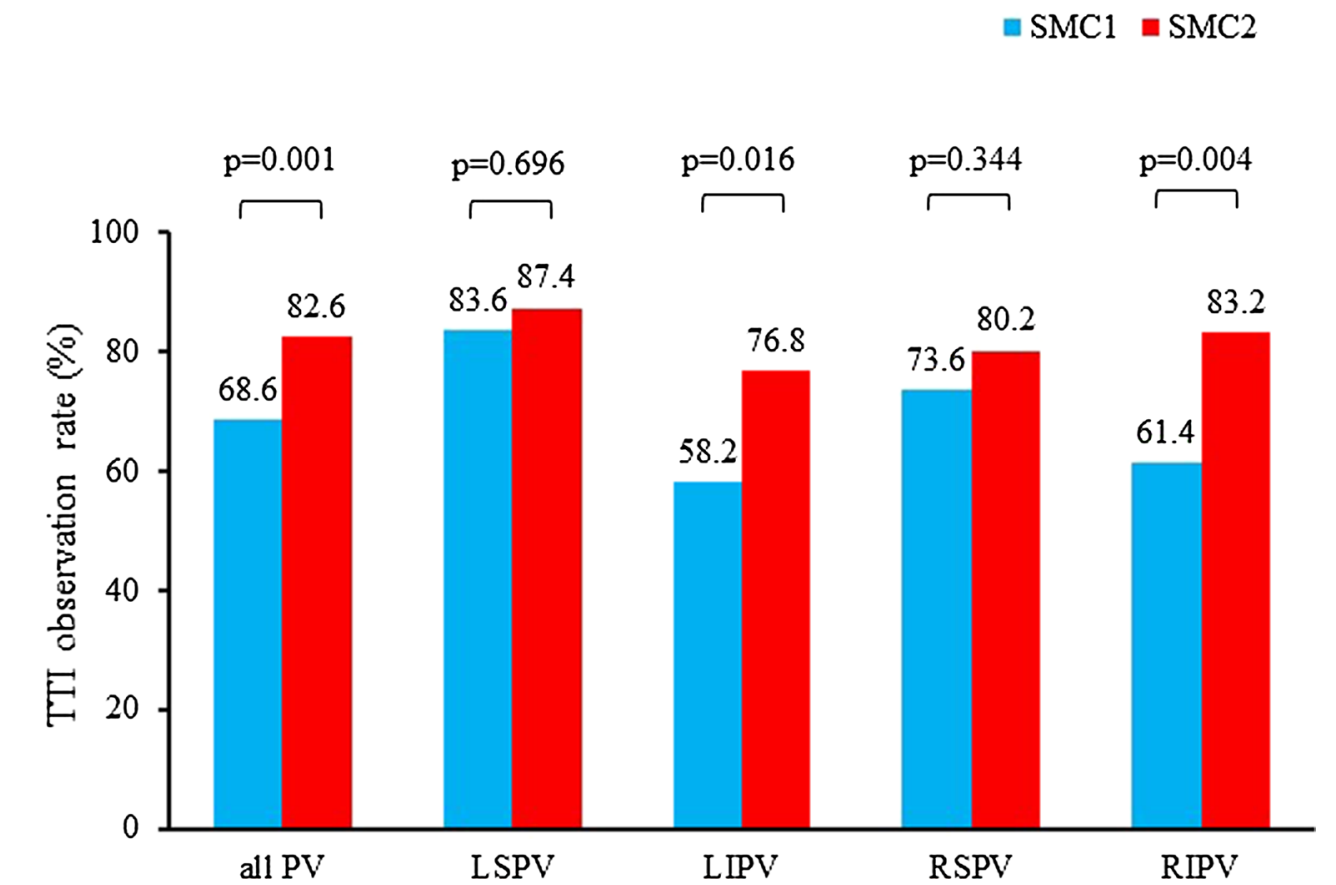


frequently in the SMC2 group compared to the control group [SMC1: 0/314 freezes (0\%) vs. SMC2: 9/655 freezes (1.4\%; $p=0.036)]$. Similarly, freeze abortion rate caused by LET $<20{ }^{\circ} \mathrm{C}$ tended to be higher when SMC2 was used although this was statistically not different ( $p=0.120$, Table 2$)$.

Remarkably, mean total freeze duration per patient was significantly longer in the SMC2 group $(17.6 \pm 5.6 \mathrm{~min})$ compared to the SMC1 group $(14.1 \pm 4.5 \mathrm{~min}, p<0.001)$. Similarly, mean number of freezes per patient was significantly higher in the SMC2 study group with $6.5 \pm 1.9$ compared to $5.5 \pm 1.5$ in the SMC1 group $(p=0.001)$.

The shaft of the SMC2 is much softer compared to SMC1, which leads to decreased maneuverability in patients with difficult PV anatomies. Specifically, the softer shaft precludes placement of the spiral tip containing the electrodes of SMC2 inside the PV in certain difficult anatomies, especially tortuous PVs (supplementary online movie 1 and 2). In these cases, backup can be increased by advancing the cryoballoon catheter over the soft shaft of the SMC2 catheter into the PV (supplementary online movie 3 and 4). Nevertheless, decreased maneuverability of SMC2 did not translate into significantly increased procedure duration or fluoroscopy time. Mean procedure duration was $72.0 \pm 18.9 \mathrm{~min}$ in the SMC1 group and 74.4 $\pm 19.1 \mathrm{~min}$ in the SMC2 group $(p=0.432)$. Mean fluoroscopy time was $15.0 \pm 5.7 \mathrm{~min}$ in the SMC1 group and $15.7 \pm 7.3 \mathrm{~min}$ in the SMC2 group $(p=0.593)$. Procedural details and acute ablation results are shown in Table 2.

\section{Complications}

Transient phrenic nerve palsy occurred in 11 patients of the whole study group with 4 PNP $(5.8 \%)$ in the SMC1 group and 7 PNP $(6.9 \%)$ in the SMC2 group $(p=1.0)$. All PNP resolved completely by the end of the procedure. No periprocedural stroke or systemic embolism occurred. Groin hematoma (without blood transfusion) was observed in one patient in the SMC1 group, whereas pericardial tamponade occurred in one patient in the SMC2 group (Table 2).

\section{Discussion}

In comparison to the $\mathrm{SMC} 1$ the novel spiral mapping catheter SMC2 is designed as a true guidewire and is available with more electrodes and with a larger diameter. How these novel features of SMC2 impact ablation characteristics during Cryo-PVI is largely unknown. First data on SMC2 used in 40 patients undergoing Cryo-PVI was reported previously [16]. However, study population and procedural data were not compared by the authors to a control group treated with SMC1.
This is the first study to report the application of the novel spiral mapping catheter SMC2 in direct comparison to SMC1 during ablation with the second generation cryoballoon. The increased spiral tip diameter of the $25 \mathrm{~mm} \mathrm{SMC2}$ together with the two additional electrodes leads to very distinct PV electrograms, which enables higher TTI observation rates. In contrast, maneuverability is decreased in SMC2 due to a soft core wire, which has been incorporated into the novel mapping catheter. This decreased maneuverability could be overcome in many cases by stabilizing the soft shaft of SMC2 by advancing the cryoballoon catheter over the shaft of the mapping catheter thereby increasing backup. Nevertheless, it would be desirable to have a stiffer variant of SMC2 at hand, probably by incorporating a stiffer core wire into the SMC2 catheter. Despite the perceived decreased maneuverability, we did not observe an increase in procedure duration or fluoroscopy time with the 2 nd generation spiral mapping catheter. However, we observed an increase in the total number of necessary cryoballoon freezes and an increase in the total freeze duration, which was mainly driven by a higher number of aborted freezes due to ineffectiveness in the SMC2 group which could be attributed to decreased stability. Taking into account that the increased TTI observation rate should have resulted in less and possibly shorter freezes, the fact that we actually observed an increase in freezes and total freeze duration might indicate, that the decreased maneuverability and stability counterbalances the advantage of higher TTI detection rates.

Real-time observation of PV isolation enables determination of the time-to-PV isolation (TTI). TTI is an independent marker of durable PV isolation or PV reconnection. Moreover, recording of the TTI enables individualization of freeze duration $[10,11,13,17]$. We have shown previously, that TTI observation rates with the 1st generation spiral mapping catheter are higher with the 3rd generation cryoballoon as compared with the 2 nd generation cryoballoon due to a shorter distal tip, which enables more proximal placement of the spiral mapping catheter within the muscular sleeve of the PV. Interestingly, with the 2nd generation spiral mapping catheter in combination with the 2 nd generation cryoballoon, we find a similar rate of observed TTI as with the 1st generation spiral mapping catheter and the 3rd generation cryoballoon $[9,13]$.

When we compared the $20 \mathrm{~mm}$ 1st and 2nd generation spiral mapping catheter to the $25 \mathrm{~mm}$ 2nd generation spiral mapping catheter, we were able to show that it is mainly the increase in diameter and probably the addition of two electrodes, that lead to increased rates of TTI detection rates. However, when we compare the $20 \mathrm{~mm} \mathrm{SMC2}$ to the $25 \mathrm{~mm}$ SMC2 we do not note a difference in TTI detections rates, but this is most likely due to the small number of procedures with the $20 \mathrm{~mm} \mathrm{SMC2}$. Both better wall contact or more proximal placement because of the bigger diameter might 
contribute to higher TTI detection rates with the $25 \mathrm{~mm} 2 \mathrm{nd}$ generation spiral mapping catheter.

\section{Conclusions}

The novel SMC2 has been designed as a true guidewire with the ability to obtain real-time PV electrograms during cryoballoon PVI. The availability of an increased diameter $(25 \mathrm{~mm})$ with 10 instead of 8 electrodes leads to a significant increase in the TTI observation rate. The softer shaft of SMC2 leads to decreased maneuverability and stability in patients with difficult anatomies. This leads to an increase in the necessary number of cryoballoon applications and freeze duration. Thus, even though one would expect that an increase in the TTI observation rate leads to a reduction in procedure duration and possibly fluoroscopy time when a TTI-based ablation protocol is used, neither procedure duration nor fluoroscopy time were decreased in the SMC2 group.

Acknowledgements We thank Beate Polte, Sylvia Dippmann, Michaela Schrade and Andrea Igel for their excellent support and Sophie Mehner, Romy Bonzheim, Tanja Kreiser, Nicole Gürtler and Isabelle Glöckler for their outstanding technical assistance. Funding was provided by Clinician Scientist Program of Ulm University Medical Center to A. Pott.

\section{Compliance with ethical standards}

Conflict of interest T Dahme received speaker's honoraria and consulting fees from Medtronic. The other authors have no conflicts to report.

Open Access This article is distributed under the terms of the Creative Commons Attribution 4.0 International License (http://creativeco mmons.org/licenses/by/4.0/), which permits unrestricted use, distribution, and reproduction in any medium, provided you give appropriate credit to the original author(s) and the source, provide a link to the Creative Commons license, and indicate if changes were made.

\section{References}

1. Packer DL, Kowal RC, Wheelan KR, Irwin JM, Champagne J, Guerra PG, Dubuc M, Reddy V, Nelson L, Holcomb RG, Lehmann JW, Ruskin JN, Cryoablation Investigators STOPAF (2013) Cryoballoon ablation of pulmonary veins for paroxysmal atrial fibrillation: first results of the north american arctic front (STOP AF) pivotal trial. J Am Coll Cardiol 61:1713-1723

2. Morillo CA, Verma A, Connolly SJ, Kuck KH, Nair GM, Champagne J, Sterns LD, Beresh H, Healey JS, Natale A, RAAFT-2 Investigators (2014) Radiofrequency ablation vs antiarrhythmic drugs as first-line treatment of paroxysmal atrial fibrillation (RAAFT-2): a randomized trial. JAMA 311:692-700

3. Kuck KH, Brugada J, Furnkranz A, Metzner A, Ouyang F, Chun KR, Elvan A, Arentz T, Bestehorn K, Pocock SJ, Albenque JP,
Tondo C, Fire and Ice Investigators (2016) Cryoballoon or radiofrequency ablation for paroxysmal atrial fibrillation. N Engl J Med 374:2235-2245

4. Miyazaki S, Kajiyama T, Watanabe T, Ichijo S, Iesaka Y (2018) Additional cryoapplications at the pulmonary vein antrum using a 28-mm second-generation cryoballoon: a pilot study of extrapulmonary vein ablation. Heart Vessels. https://doi.org/10.1007/ s00380-018-1142-4

5. Shah D (2009) Electrophysiological evaluation of pulmonary vein isolation. Europace 11:1423-1433

6. Aryana A, Mugnai G, Singh SM, Pujara DK, de Asmundis C, Singh SK, Bowers MR, Brugada P, d'Avila A, O'Neill PG, Chierchia GB (2016) Procedural and biophysical indicators of durable pulmonary vein isolation during cryoballoon ablation of atrial fibrillation. Heart Rhythm 13:424-432

7. Ciconte G, Mugnai G, Sieira J, Velagic V, Saitoh Y, Irfan G, Hunuk B, Stroker E, Conte G, Di Giovanni G, Baltogiannis G, Wauters K, Brugada P, de Asmundis C, Chierchia GB (2015) On the quest for the best freeze: predictors of late pulmonary vein reconnections after second-generation cryoballoon ablation. Circ Arrhythm Electrophysiol 8:1359-1365

8. Ciconte G, Velagic V, Mugnai G, Saitoh Y, Irfan G, Hunuk B, Stroker E, Conte G, Sieira J, Di Giovanni G, Baltogiannis G, Brugada P, de Asmundis C, Chierchia GB (2016) Electrophysiological findings following pulmonary vein isolation using radiofrequency catheter guided by contact-force and second-generation cryoballoon: lessons from repeat ablation procedures. Europace 18:71-77

9. Pott A, Petscher K, Messemer M, Rottbauer W, Dahme T (2016) Increased rate of observed real-time pulmonary vein isolation with third-generation short-tip cryoballoon. J Interv Card Electrophysiol 47:333-339

10. Chun KR, Stich M, Furnkranz A, Bordignon S, Perrotta L, Dugo D, Bologna F, Schmidt B (2017) Individualized cryoballoon energy pulmonary vein isolation guided by real-time pulmonary vein recordings, the randomized ICE-T trial. Heart Rhythm 14:495-500

11. Aryana A, Kenigsberg DN, Kowalski M, Koo CH, Lim HW, O'Neill PG, Bowers MR, Hokanson RB, Ellenbogen KA, CryoDOSING Investigators (2017) Verification of a novel atrial fibrillation cryoablation dosing algorithm guided by time-to-pulmonary vein isolation: results from the cryo-DOSING study (cryoballoonablation DOSING based on the assessment of time-to-effect and pulmonary vein isolation guidance). Heart Rhythm 14:1319-1325

12. Ferrero-de-Loma-Osorio A, Garcia-Fernandez A, Castillo-Castillo J, Izquierdo-de-Francisco M, Ibanez-Criado A, Moreno-Arribas J, Martinez A, Bertomeu-Gonzalez V, Lopez-Mases P, AjoFerrer M, Nunez C, Bondanza-Saavedra L, Sanchez-Gomez JM, Martinez-Martinez JG, Chorro-Gasco FJ, Ruiz-Granell R (2017) Time-to-effect-based dosing strategy for cryoballoon ablation in patients with paroxysmal atrial fibrillation: results of the plusONE multicenter randomized controlled noninferiority trial. Circ Arrhythm Electrophysiol. https://doi.org/10.1161/CIRCE P.117.005318

13. Pott A, Kraft C, Stephan T, Petscher K, Rottbauer W, Dahme $T$ (2018) Time-to-isolation guided titration of freeze duration in 3rd generation short-tip cryoballoon pulmonary vein isolationcomparable clinical outcome and shorter procedure duration. Int J Cardiol 255:80-84

14. Pott A, Messemer M, Petscher K, Iturbe-Orbe M, Bothner C, Rottbauer W, Dahme T (2017) Clinical outcome of 2nd generation cryoballoon pulmonary vein isolation in patients over 75 years of age. J Cardiol 69:24-29

15. Calkins H, Hindricks G, Cappato R, Kim YH, Saad EB, Aguinaga L, Akar JG, Badhwar V, Brugada J, Camm J, Chen PS, Chen SA, Chung MK, Nielsen JC, Curtis AB, Wyn Davies D, Day JD, 
d'Avila A, de Groot NMSN, Di Biase L, Duytschaever M, Edgerton JR, Ellenbogen KA, Ellinor PT, Ernst S, Fenelon G, Gerstenfeld EP, Haines DE, Haissaguerre M, Helm RH, Hylek E, Jackman WM, Jalife J, Kalman JM, Kautzner J, Kottkamp H, Kuck KH, Kumagai K, Lee R, Lewalter T, Lindsay BD, Macle L, Mansour M, Marchlinski FE, Michaud GF, Nakagawa H, Natale A, Nattel S, Okumura K, Packer D, Pokushalov E, Reynolds MR, Sanders $\mathrm{P}$, Scanavacca M, Schilling R, Tondo C, Tsao HM, Verma A, Wilber DJ, Yamane T (2017) 2017 HRS/EHRA/ECAS/APHRS/ SOLAECE expert consensus statement on catheter and surgical ablation of atrial fibrillation: executive summary. J Interv Card Electrophysiol 50:1-55
16. Reissmann B, Schluter M, Santoro F, Maurer T, Heeger CH, Lemes C, Fink T, Riedl J, Rillig A, Mathew S, Ouyang F, Kuck KH, Metzner A (2018) Does size matter? Cryoballoon-based pulmonary vein isolation using a novel $25-\mathrm{mm}$ circular mapping catheter. Circ J 82:666-671

17. Dorwarth U, Schmidt M, Wankerl M, Krieg J, Straube F, Hoffmann E (2011) Pulmonary vein electrophysiology during cryoballoon ablation as a predictor for procedural success. J Interv Card Electrophysiol 32:205-211 